\title{
Numerical Simulation and Analysis of Supersonic flow over a flat plate
}

\author{
${ }^{1}$ Rupesh R. P. Koirala, ${ }^{2}$ K. V. S. Santosh Kumar, ${ }^{3}$ Sanjay Adhikari, \\ ${ }^{4}$ Dharne Praveen \\ National Institute of Technology, Kurukshetra
}

\begin{abstract}
Two dimensional supersonic flows over a sharp flat plate at zero incidence has been investigated numerically. A fictitious curvature is formed due to the development of a boundary layer at the leading edge of the plate. The pressure along the entire surface and at the trailing edge has been computed over a wide range of Mach numbers and two different temperatures. The temperature variation due to dissipation of kinetic energy within the boundary layer has been analysed numerically.

Keywords: Computational Fluid Dynamics, Supersonic Flow, Flat Plate, Numerical Investigation.
\end{abstract}

\section{Introduction}

The region between the surface and the shock is called the shock layer. Depending on (for example) Mach number, Reynolds number and surface temperature the shock layer can be characterized by the region of viscous flow and inviscid flow (refer to Fig.1), or the entire layer can be fully viscous, a so called merged shock layer (refer to Fig.2). Furthermore, dissipation of kinetic energy within the boundary layer (viscous dissipation) can cause high flow field temperatures and thus high heat transfer rates.

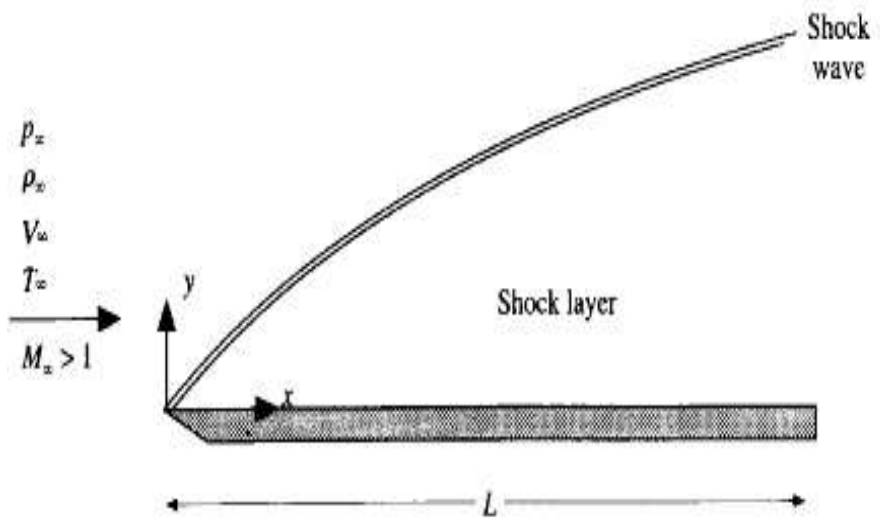

Figure 1 : Supersonic flow over a sharp leading-edged flat plate at zero incidence.

\section{Governing Equations}

Time dependent Navier-Stokes equations have been used to evolve to the correct steady-state solution. The governing flow equations take the form:

Continuity:

$\frac{\partial \rho}{\partial t}+\frac{\partial}{\partial x}(\rho u)+\frac{\partial}{\partial y}(\rho v)=0$

X Momentum:

$$
\frac{\partial}{\partial t}(\rho u)+\frac{\partial}{\partial x}\left(\rho u^{2}+p-\tau_{x x}\right)+\frac{\partial}{\partial y}\left(\rho u v-\tau_{y x}\right)=0
$$




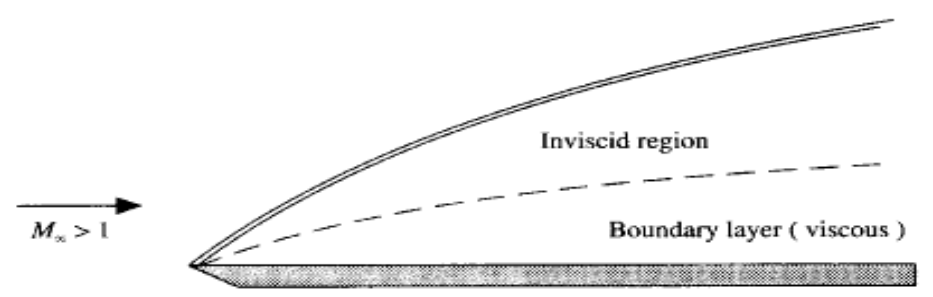

(a)

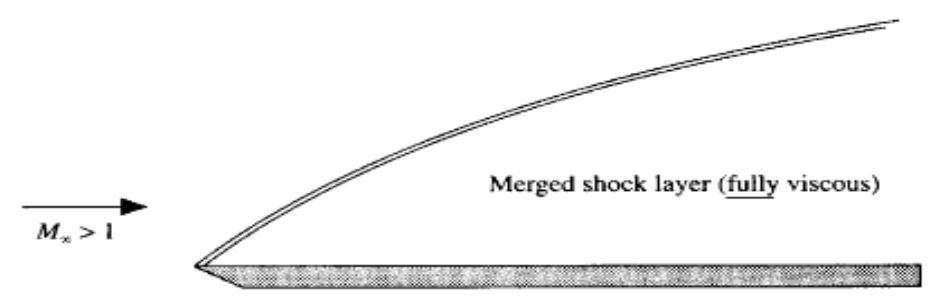

(b)

Figure 2 : (a) Supersonic flow over a flat plate with a distinct boundary layer and region of inviscid flow. (b) Supersonic flow over a flat plate with a merged shock layer.

Y Momentum:

$\frac{\partial}{\partial t}(\rho v)+\frac{\partial}{\partial x}\left(\rho u v-\tau_{x y}\right)+\frac{\partial}{\partial y}\left(\rho v^{2}+p-\tau_{y y}\right)=0$

Energy:

$\frac{\partial}{\partial t}\left(E_{t}\right)+\frac{\partial}{\partial x}\left[\left(E_{t}+p\right) u+q_{x}-u \tau_{x x}-v \tau_{x y}\right]+\frac{\partial}{\partial y}\left[\left(E_{t}+p\right) v+q_{y}-u \tau_{y x}-v \tau_{y y}\right]=0$

Where $\mathrm{t}, \mathrm{x}$ and $\mathrm{y}$ are the time, $\mathrm{x}$ and $\mathrm{y}$ coordinates. $\rho, \mathrm{u}, \mathrm{v}, \mathrm{p}$ are density, velocity in $\mathrm{x}$ direction, velocity in $\mathrm{y}$ direction and pressure respectively. $E_{t}$ is the sum of kinetic energy and internal energy and $E_{t}=\rho\left(e+\frac{V^{2}}{2}\right)$. $\tau_{x y}, \tau_{x x}, \tau_{y y}$ are shear stresses where

Where $\mu$ is dynamic viscosity.

$$
\begin{gathered}
\tau_{x y}=\mu\left(\frac{\partial u}{\partial y}+\frac{\partial v}{\partial x}\right) \\
\tau_{x x}=\lambda(\nabla \cdot V)+2 \mu \frac{\partial u}{\partial x} \\
\tau_{y y}=\lambda(\nabla \cdot V)+2 \mu \frac{\partial v}{\partial y} \\
q_{x}=-k \frac{\partial T}{\partial x} \\
q_{y}=-k \frac{\partial T}{\partial y}
\end{gathered}
$$

This forms a system of four basic equations with nine unknown variables. For solving these equations, five additional equations used are the equation of state for a perfect gas (Ref. [1]), calorific equation of state (Ref. [1]), Sutherland's law for a calorifically perfect gas (Ref. [2]), resultant velocity equation and the relationship between Prandtl number and viscosity (Ref. [2]) has been used.

Free stream conditions are imposed on the front end of the flat plate. No slip situation is enforced on the flat plate and its temperature is assumed to be constant. 


\section{Results}

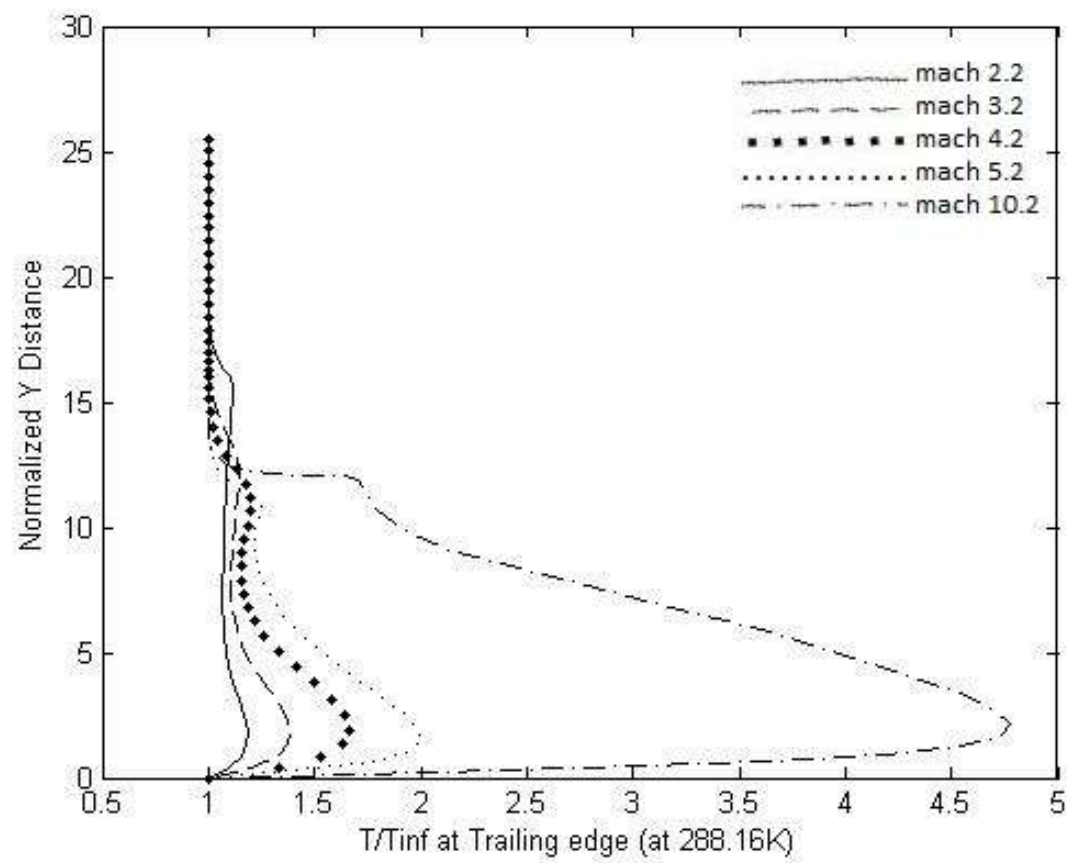

Figure 3 : Variation of normalised temperature at the trailing edge for different Mach numbers for a plate surface temperature of $288.16 \mathrm{~K}$.

The normalized temperature profile at the trailing edge of the flat plate at $288.16 \mathrm{~K}$ for different mach numbers is computed. It is seen that the temperature of the flow field increases at the boundary layer due to viscous dissipation near the plate surface. The increase in temperature is higher for higher mach numbers (i.e. with increasing Reynolds number). The temperature of the flow at the trailing edge becomes equal to the ambient temperature for all mach numbers past the boundary layers.

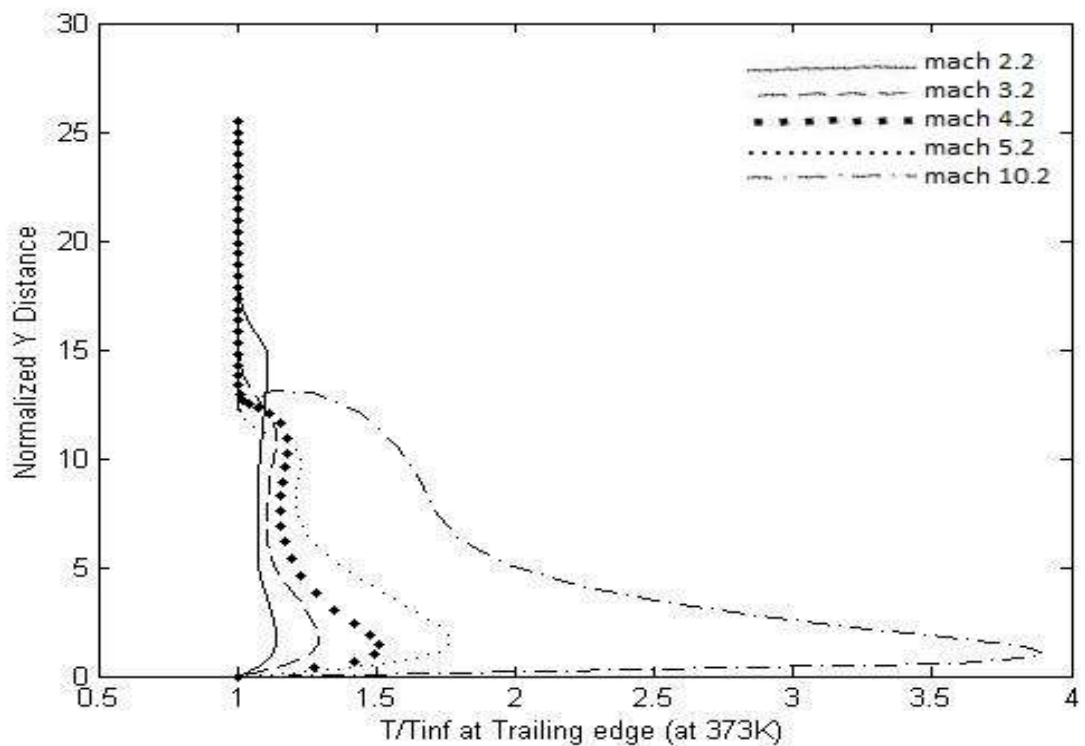

Figure 4 : Variation of normalised temperature at the trailing edge for different Mach numbers for a plate surface temperature of $373 \mathrm{~K}$.

The normalized temperature profile at the trailing edge of the flat plate at $373 \mathrm{~K}$ for different mach numbers is computed. It has been observed that the increase in the temperature of the flow field at the trailing edge is lower compared to the flow at $288.16 \mathrm{~K}$. Again the increase in temperature is higher for higher mach numbers (i.e. with increasing Reynolds number; however the Reynolds number for $373 \mathrm{~K}$ is lower than for $288.16 \mathrm{~K}$ for all other similar conditions). Here also the temperature of the flow at the trailing edge becomes equal to the ambient temperature for all mach numbers past the boundary layers. 


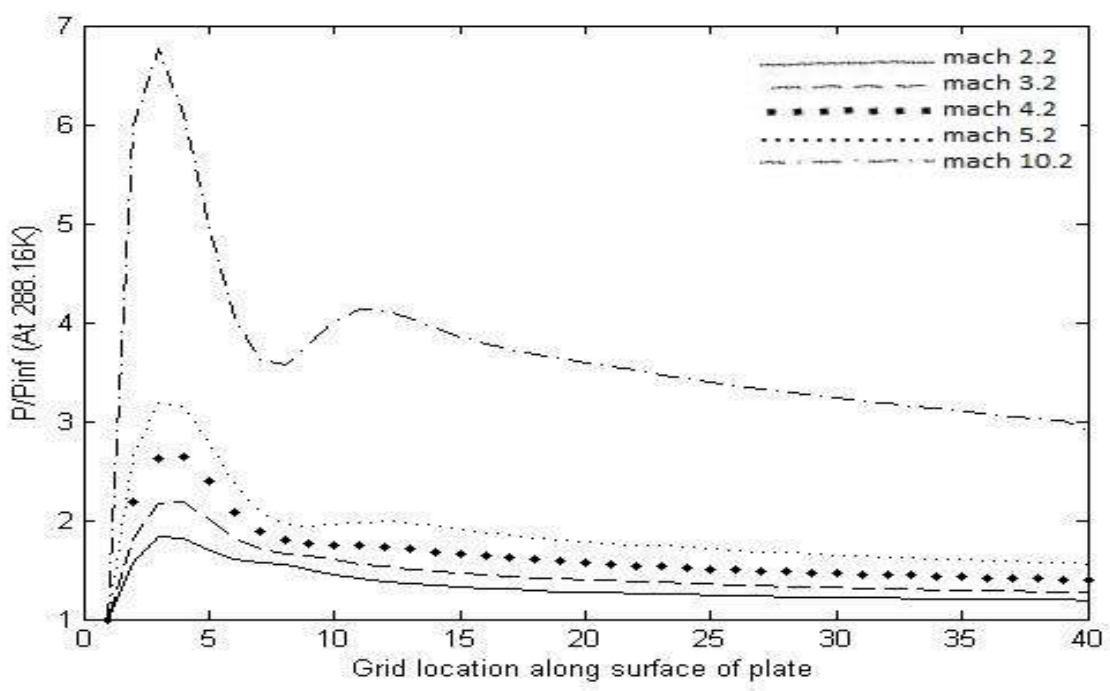

Figure 5 : Variation of normalised surface pressure at 288.16K for different Mach numbers.

Here, normalized surface pressure distribution is plotted as a function of distance from the leading edge. Initially oscillations are observed showing higher increase in pressure in the region near the leading edge. However, the oscillations disappear past the leading edge and a stable result is obtained. With the increase in Mach number, there is consequent increase in the non dimensional pressure, with the peak pressure for mach 10.2 at $288.16 \mathrm{~K}$ surface temperature (Reynolds number=2376.5) being 6.7657 times the ambient pressure (Pinf). At the trailing edge, the pressure is 2.9045 times that of the ambient pressure.

Results obtained for the computation of normalised surface pressure distribution at $373 \mathrm{~K}$ for same mach numbers as in the case of $288.16 \mathrm{~K}$ plate surface temperature have been compared. It was observed that the pressure at the trailing edge for a mach number of $10.2(\mathrm{Re}=1512.3)$ is 2.6623 times the ambient pressure.

The pressure in both the cases is gradually decreasing along the plate surface from the leading edge to the trailing edge of the plate.

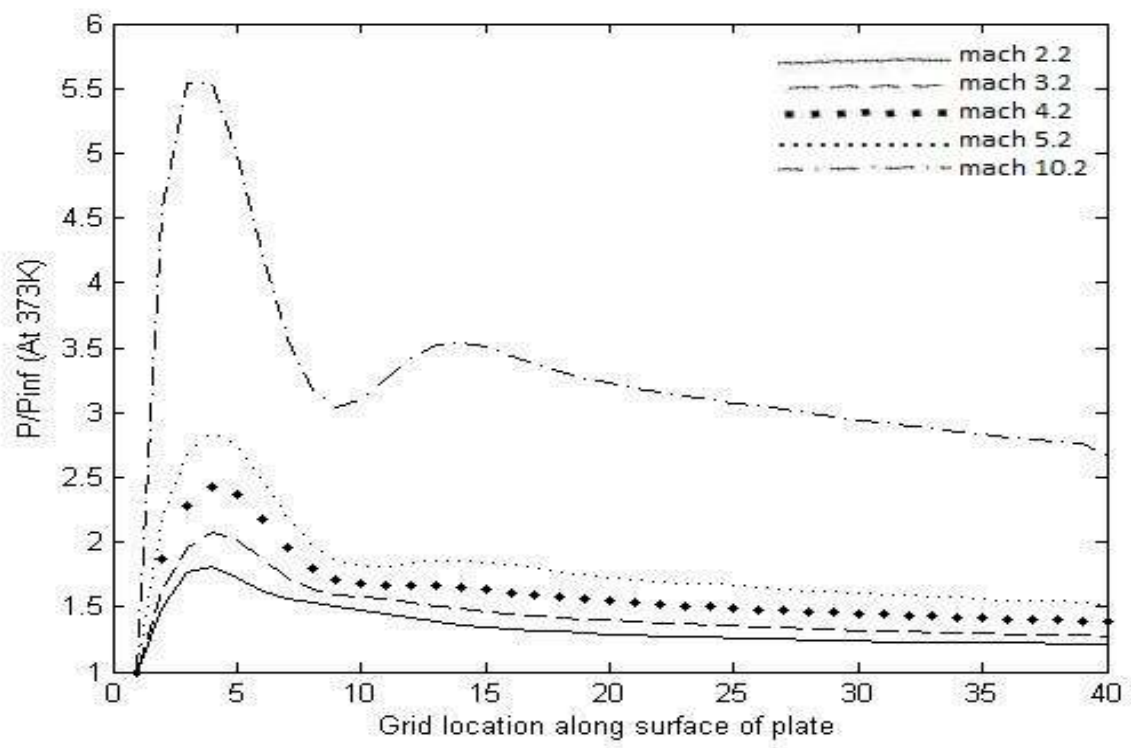

Figure 6 : Variation of normalised surface pressure at 373K for different Mach numbers. 


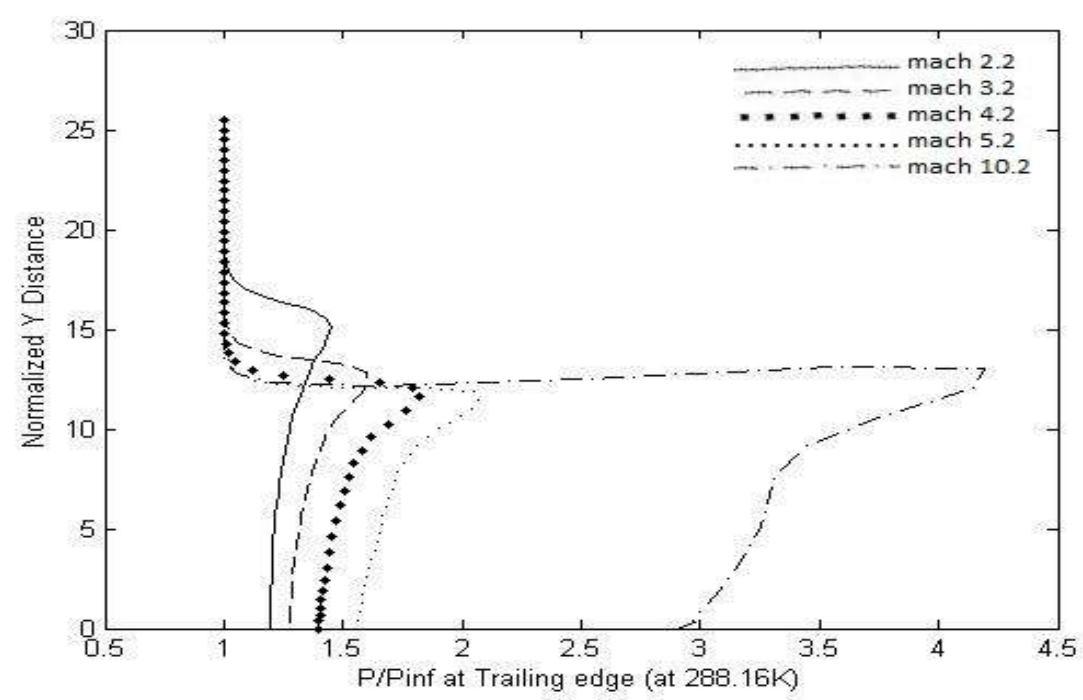

Figure 7 : Variation of normalised pressure at $288.16 \mathrm{~K}$ at the trailing edge for different Mach numbers.

The pressure distribution in the entire flow field has been computed. The normalised pressure distribution at the trailing edge for the inflow velocity with different mach numbers is plotted( at $288.16 \mathrm{~K}$ and at $373 \mathrm{~K})$. It has been observed that due to the formation of boundary layer, the flow velocity decreases and hence pressure increases as shown by the plot for different mach numbers at the trailing edge. With the increase in the mach numbers, the non dimensional pressure is also increasing at the trailing edge. Comparing the result at $288.16 \mathrm{~K}$ with the one obtained at $373 \mathrm{~K}$, it has been observed that the non dimensional peak pressure decreases for increasing temperatures. For a plate temperature of $288.16 \mathrm{~K}$, these ratios were found to be 4.1927 at $10.2 \mathrm{mach}, 2.0796$ at $5.2 \mathrm{mach}$ and 1.4484 at $2.2 \mathrm{mach}$. Similarly for a plate temperature of $373 \mathrm{~K}$, these ratios were found to be 3.7024 at $10.2 \mathrm{mach}, 1.9615$ at $5.2 \mathrm{mach}$, and 1.4282 at $2.2 \mathrm{mach}$. Hence it can be inferred that the ratio is less for the same Mach number at higher temperature.

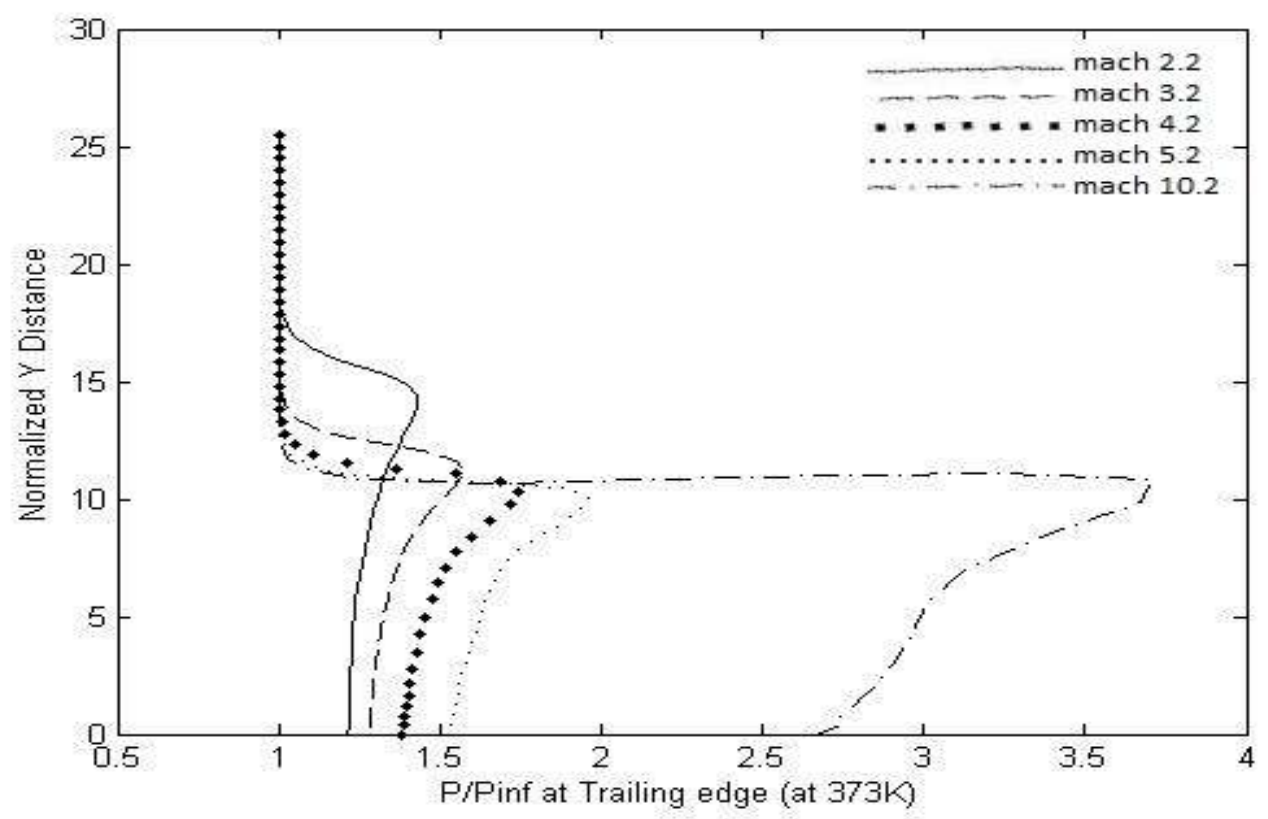

Figure 8 : Variation of normalised pressure at $373 \mathrm{~K}$ at the trailing edge for different Mach numbers. 


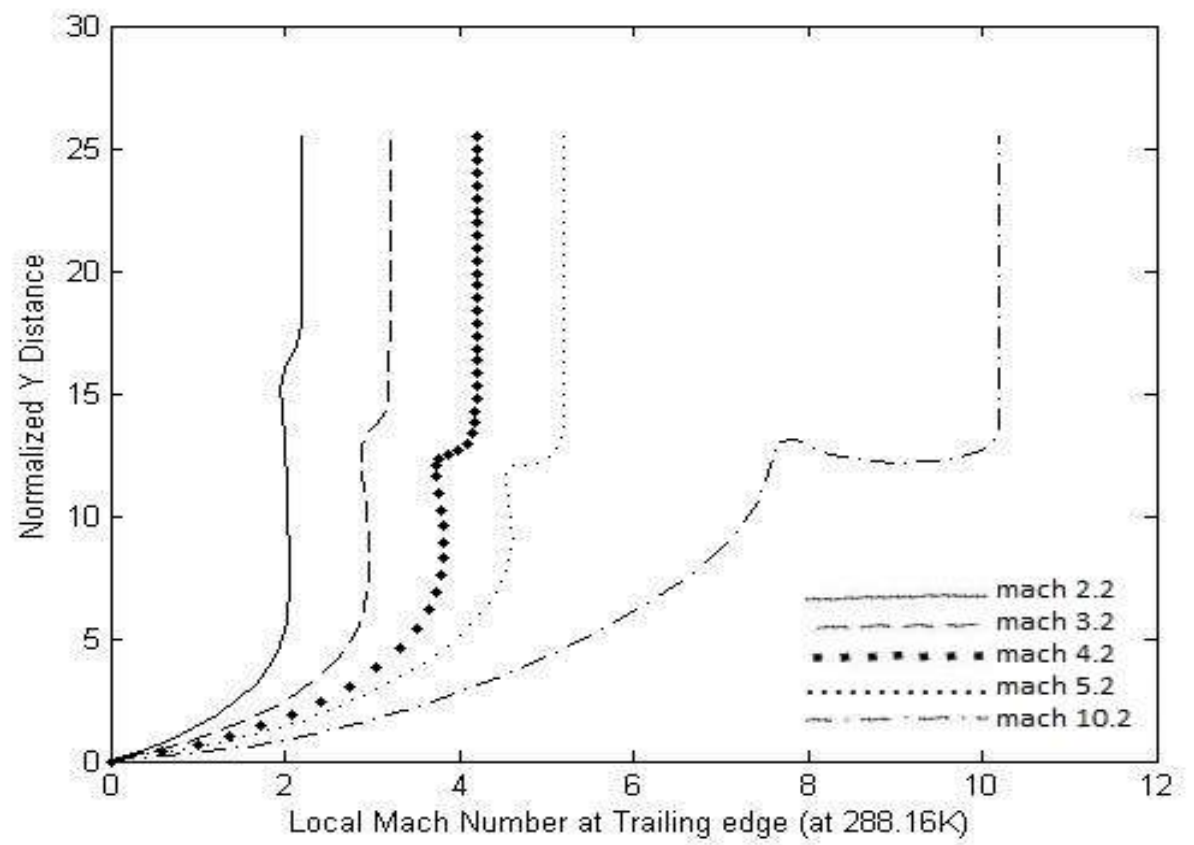

Figure 9 : Variation of Mach number at the trailing edge for a plate surface temperature of 288.16K.

During the numerical experiment, Mach number variations for the different initial mach numbers at temperature 288.16K were also examined numerically. It was also found that due to the formation of the boundary layer above the plate, the Mach number decreases and gradually increases along the vertical height and suddenly attains initial value of the Mach number past the boundary layer.

\section{Conclusion}

In this study we have analysed the following things, i.e., variation of normalised temperature at the trailing edge at different surface temperatures and mach numbers, variation of surface pressure at different surface temperatures and mach numbers along with the variation of normalised pressure at the trailing edge at different surface temperatures and mach numbers. We can see that these parameters have a deep impact on the nature of curves but their basic behaviour remains the same. For further study we can keep the wall adiabatic instead of the current assumption of an isothermal wall.

\section{Acknowledgment}

We thank Dr. P K Saini (Associate Professor, NIT Kurukshetra) for his constant guidance, support and motivation during the course of our research. We thank all the members of the Department of Mechanical Engineering at NIT Kurukshetra who has supported us in attaining these results.

\section{References}

[1] John D. Anderson, Jr.: Computational Fluid Dynamics, McGraw-Hill, Inc.

[2] John D. Anderson, Jr.: Fundamentals of Aerodynamics, McGraw-Hill, Inc.

[3] John D. Anderson, Jr.: Hypersonic and High Temperature Gas Dynamics, McGraw-Hill, Inc.

[4] R. W. MacCormack, “A Numerical Approach for solving equations of compressible viscous flow,” AIAA J. 20, 1275 (1982).

[5] Schichting, H.: Boundary Layer Theory, McGraw-Hill, Inc.

[6] Patankar, S. V.: Numerical Heat Transfer and Fluid Flow, McGraw-Hill, Inc.

[7] Fletcher, C.A.: Computational Techniques for Fluid Dynamics, Springer-Verlag, Berlin, 1988

[8] T. J. Chung, Computational Fluid Dynamics, Cambridge. 